DESY 94-124

ISSN 0418-9833

hep-ph/9407347

July 1994

\title{
Next-to-Leading Order Fragmentation Functions for Pions and Kaons
}

\author{
J. Binnewies, B.A. Kniehl, and G. Kramer \\ II. Institut für Theoretische Physik, Universität Hamburg \\ Luruper Chaussee 149, 22761 Hamburg, Germany
}

\begin{abstract}
We present new sets of fragmentation functions for charged pions and kaons, both at leading and next-to-leading order. They are fitted to TPC data taken at energy $\sqrt{s}=29 \mathrm{GeV}$ and describe excellently a wealth of other $e^{+} e^{-}$data on charged-hadron production, ranging from $\sqrt{s}=5.2 \mathrm{GeV}$ way up to LEP 1 energy. They also agree with data on the production of neutral pions and kaons, if one makes the natural assumption that the respective fragmentation functions are related to the charged counterparts by $\mathrm{SU}(2)$ symmetry. We also list simple parameterizations of the $x$ and $Q^{2}$ dependence of our results, which may be implemented conveniently in applications.
\end{abstract}

*Supported by Bundesministerium für Forschung und Technologie, Bonn, Germany, under Contract $056 \mathrm{HH}$ 93P (5) and by EEC Program Human Capital and Mobility through Network Physics at High Energy Colliders under Contract CHRX-CT93-0357 (DG12 COMA). 


\section{Introduction}

The Lagrangian of quantum chromodynamics contains quarks and gluons as elementary fields. Allowing for these particles to appear as asymptotic states, we can evaluate scattering amplitudes perturbatively, in principle to arbitrary precision. Of course, this picture violates the principle of confinement of colour; experiments detect hadrons rather than quarks and gluons. Nevertheless, this simplified computational procedure is very successful in describing the production of jets of hadrons at high transverse momenta $\left(p_{T}\right)$ in scattering processes and at high centre-of-mass (c.m.) energies $(\sqrt{s})$ in $e^{+} e^{-}$annihilation. Apparently, clustering partons in the final state yields a valid approximation, although this does not account for any details of hadronization. On the other hand, experiments are providing us with copious information on inclusive production of single hadrons, which cannot be interpreted along these lines. In this case, we need a concept of describing how partons turn into hadrons. In the framework of the QCD-improved parton model, this is achieved by introducing fragmentation functions, $D_{a}^{h}\left(x, Q^{2}\right)$. Their values correspond to the probability that a parton, $a$, which is produced at short distance, $\sqrt{1 / Q^{2}}$, fragments into a hadron, $h$, carrying a fraction, $x$, of the momentum of $a$. Unfortunately, it is not yet understood how these functions can be derived from the QCD Lagrangian, in particular for hadrons with masses comparable to the QCD asymptotic scale parameter, $\Lambda$. However, given their $x$ dependence at some scale $Q_{0}^{2}$, the evolution with $Q^{2}$ may be computed perturbatively in QCD. This allows us to test QCD quantitatively within one experiment observing single hadrons at different values of $p_{T}$ (in the case of scattering) or taking data at different values of $\sqrt{s}$ (in the case of $e^{+} e^{-}$annihilation). Moreover, appealing to the factorization theorem, we may assume that the $D_{a}^{h}\left(x, Q^{2}\right)$ functions are independent of the process in which they have been determined, and characterize a universal property of $h$. This enables us to make quantitative predictions for other experiments, too.

Recently, precise data on inclusive hadron production at $p \bar{p}, e p$, and $e^{+} e^{-}$colliders have become available. A comprehensive review of inclusive particle spectra in earlier $e^{+} e^{-}$ experiments may be found in [1]. In a series of papers, two of us-partly in collaboration 
with Borzumati-have confronted $p \bar{p}$ data by CDF and UA2 and ep data by H1 on pion and kaon production with theoretical predictions at NLO [2]3]. Although we found reasonable agreement, we have to bear in mind that a possible limitation of our analyses is related to the point that the literature on pion and kaon fragmentation functions is now rather aged and, perhaps, slightly obsolete. The most recent sets we could locate were the ones by Baier et al. [四, which were updated and complemented by Anselmino et al. [5]. These sets are LO fits to low-energy $e^{+} e^{-}$and deep-inelastic muon-nucleon data.

Recently, NLO sets for neutral pions were presented in [6]. These were fitted partly to $e^{+} e^{-}$data in the $30 \mathrm{GeV}$ range, partly to fixed-target and collider data, with $23 \mathrm{GeV} \leq$ $\sqrt{s} \leq 630 \mathrm{GeV}$, and partly to data of $e^{+} e^{-}$annihilation at $30 \mathrm{GeV}$ simulated using a Monte Carlo event generator. Unfortunately, only the $x$ distributions at $Q_{0}^{2}$ are given in [6]; an implementation of the $Q^{2}$ dependence is not available. New NLO sets have also been constructed for the eta meson and charged pions just recently [7], again using Monte Carlo data. Also here, the $Q^{2}$ dependence is not given.

Since our goal is to test quantitatively the QCD-improved parton model, we consider it requisite to perform an independent analysis based on real experimental information. Complementarily to [6], we shall focus attention on charged pions and kaons, averaged separately. Similarly to [6], we analyze $e^{+} e^{-}$data at around $30 \mathrm{GeV}$. A different avenue was taken in $[\mathbb{8}$, where charged-hadron fragmentation functions were extracted from LEP 1 data, not distinguishing between the various particle species.

The organization of this paper is as follows. In Sect. 2, we shall introduce the formalism needed to extract fragmentation functions from $e^{+} e^{-}$data on inclusive hadron production at NLO. In Sect. 3, we shall carry out the actual analysis and discuss our results. In Sect. 4, we shall check our results against data on neutral-pion and -kaon production as well as data on charged-hadron production without separation of the particle species. We shall also compare our results with those of [8]. Our conclusions will be summarized in Sect. 5 . In the Appendix, we shall list simple parameterizations of our sets and quote their range of validity. 


\section{Formalism}

The inclusive production of a hadron, $h$, by $e^{+} e^{-}$annihilation,

$$
e^{+} e^{-} \rightarrow(\gamma, Z) \rightarrow h+X
$$

is completely characterized by three observables. These are the energy fraction of the outgoing hadron,

$$
x=\frac{2 E_{h}}{\sqrt{s}}
$$

its angle with the beam axis, $\theta$, and the total energy of the system, $\sqrt{s}$. For massless hadrons, definition (2) agrees with the convention used in experiments, whereas for nonvanishing masses, $m_{h}$, one has to adjust the theoretical prediction by including a factor

$\beta_{h}=\sqrt{1-m_{h}^{2} / E_{h}^{2}}$. Choice of reference frame is not an issue here, since, at $e^{+} e^{-}$colliders, the laboratory and c.m. frames coincide. The cross section of (1) exhibits the angular structure

$$
\frac{d^{2} \sigma\left(e^{+} e^{-} \rightarrow h+X\right)}{d x d \cos \theta}=\frac{3}{8}\left(1+\cos ^{2} \theta\right) \frac{d \sigma^{T}}{d x}+\frac{3}{4} \sin ^{2} \theta \frac{d \sigma^{L}}{d x}+\frac{3}{4} \cos \theta \frac{d \sigma^{A}}{d x}
$$

where the superscripts $T$ and $L$ denote the contributions due to transverse and longitudinal polarizations, respectively, and the asymmetric term, labelled $A$, accounts for the interference of the photon with the $Z$ boson. Usually, experiments do not determine $\theta$ distributions. Thus, we shall integrate over $\theta$. This eliminates the asymmetric term in (3).

The partonic subprocesses may be treated exactly in perturbative QCD. However, this is not yet possible for the process of hadronization. As explained in the Introduction, the latter is described in terms of phenomenological fragmentation functions, which must be extracted from experiment. In the language of the QCD-improved parton model, the $x$ distribution of (11) emerges from the $x$ distribution of $e^{+} e^{-} \rightarrow a+X,\left(d \sigma_{a} / d x\right)\left(x, \mu^{2}, Q^{2}\right)$, through convolution with $D_{a}^{h}\left(x, Q^{2}\right)$,

$$
\frac{s}{\beta_{h}} \frac{d \sigma\left(e^{+} e^{-} \rightarrow h+X\right)}{d x}=\sum_{a} \int_{x}^{1} \frac{d z}{z} D_{a}^{h}\left(z, M_{f}^{2}\right) s \frac{d \sigma_{a}}{d y}\left(\frac{x}{z}, \mu^{2}, M_{f}^{2}\right) .
$$


Here, the sum extents over all active partons $(a=g, u, d, s, c, b), \mu$ is the renormalization scale of the partonic subprocess, and $M_{f}$ is the so-called fragmentation scale, which corresponds to the typical energy where the transition from the perturbative to the nonperturbative regime takes place. In next-to-leading order (NLO), $M_{f}$ defines the point where the divergence associated with collinear radiation off parton $a$ is to be subtracted. As usual, we work in the massless-quark approximation, i.e., we neglect threshold effects. As a consequence, the bottom quark is taken to be active throughout the entire $Q^{2}$ evolution and to contribute to the cross section also at low energies. We checked that this slight inconsistency is inconsequential for the goodness of our fits. To NLO in the $\overline{\mathrm{MS}}$ scheme, the cross sections of the relevant subprocesses are given by [9]

$$
\begin{aligned}
\frac{d \sigma_{q_{i}}}{d y}\left(y, \mu^{2}, M_{f}^{2}\right) & =\sigma_{0} N_{c} e_{q_{i}}^{2}\left[\delta(1-y)+\frac{\alpha_{S}\left(\mu^{2}\right)}{2 \pi}\left(P_{q q}^{(0, T)}(y) \ln \frac{s}{M_{f}^{2}}+K_{q}^{T}(y)+K_{q}^{L}(y)\right)\right], \\
\frac{d \sigma_{g}}{d y}\left(y, \mu^{2}, M_{f}^{2}\right) & =\sigma_{0} N_{c}\left(\sum_{i=1}^{2 N_{f}} e_{q_{i}}^{2}\right) \frac{\alpha_{S}\left(\mu^{2}\right)}{2 \pi}\left(P_{q g}^{(0, T)}(y) \ln \frac{s}{M_{f}^{2}}+K_{g}^{T}(y)+K_{g}^{L}(y)\right),
\end{aligned}
$$

where $\sigma_{0}=\left(4 \pi \alpha_{\mathrm{QED}}^{2} / 3 s\right)$ is the total cross section of $e^{+} e^{-} \rightarrow \mu^{+} \mu^{-}$for massless leptons, $N_{c}=3, e_{q_{i}}$ is the electric charge of quark $q_{i}$ in units of the positron charge, $P_{a b}^{(0, T)}(y)$ is the LO term of the transverse $a \rightarrow b$ splitting function,

$$
P_{a b}^{T}\left(y, \alpha_{S}\left(Q^{2}\right)\right)=P_{a b}^{(0, T)}(y)+\frac{\alpha_{S}\left(Q^{2}\right)}{2 \pi} P_{a b}^{(1, T)}(y)+\cdots,
$$

and

$$
\begin{aligned}
K_{q}^{T}(y)= & C_{F}\left[\frac{3}{2}(1-y)-\frac{3}{2}\left(\frac{1}{1-y}\right)_{+}+2 \frac{1+y^{2}}{1-y} \ln y\right. \\
& \left.+\left(1+y^{2}\right)\left(\frac{\ln (1-y)}{1-y}\right)_{+}+\left(\frac{2 \pi^{2}}{3}-\frac{9}{2}\right) \delta(1-y)\right], \\
K_{q}^{L}(y)= & C_{F}, \\
K_{g}^{T}(y)= & C_{F}\left[\frac{1+(1-y)^{2}}{y}(\ln (1-y)+2 \ln y)-2 \frac{1-y}{y}\right], \\
K_{g}^{L}(y)= & 2 C_{F} \frac{1-y}{y}
\end{aligned}
$$

with $C_{F}=\left(N_{c}^{2}-1\right) /\left(2 N_{c}\right)=4 / 3$. As usual, the subscript + denotes distributions. The NLO formula of $\alpha_{s}(\mu)$ may be found, e.g., in [10]. Throughout this work, we choose 
$\Lambda=190 \mathrm{MeV}$, in order to be compatible with recent sets of parton density functions for the proton and photon. The scales $\mu$ and $M_{f}$ are identified with the only intrinsic energy scale, $\sqrt{s}$. For this choice of scales, the logarithmic terms in (可) involving $s$ vanish, so that the NLO corrections are expressed just in terms of the $K$ functions. We observe that $K_{g}^{T}(y)+K_{g}^{L}(y)<0$ for any value of $y$, so that the gluon contributes destructively to (荬).

Having defined the partonic subprocesses, we turn to the fragmentation functions. Their $x$ distributions are not yet calculable in the framework of perturbative QCD. However, once we know them at some scale $Q_{0}^{2}$, the $Q^{2}$ evolution is determined by the DGLAP equations [11]. Our task is thus to construct a model for the $x$ distributions at a starting scale $Q_{0}^{2}$, which, after evolution, fits the data at scale $Q^{2}$. The DGLAP equations read

$$
\frac{d}{d \ln Q^{2}} D_{a}^{h}\left(x, Q^{2}\right)=\frac{\alpha_{S}\left(Q^{2}\right)}{2 \pi} \sum_{b} \int_{x}^{1} \frac{d y}{y} P_{b a}^{T}\left(y, \alpha_{S}\left(Q^{2}\right)\right) D_{b}^{h}\left(\frac{x}{y}, Q^{2}\right) .
$$

That is, we have to solve a system of integro-differential equations. This may be achieved with the help of the Mellin-transform technique [12]. The Mellin transform, F, of a function, $f$, is defined as the set of its moments,

$$
F(n)=\int_{0}^{1} d x x^{n-1} f(x) .
$$

The inverse transformation is

$$
f(x)=\frac{1}{2 \pi i} \int_{\gamma-i \infty}^{\gamma+i \infty} d n x^{-n} F(n),
$$

where the path of integration must be chosen to the right of all singularities of the integrand. In our numerical analysis, we choose $\gamma=10$. The essential property of this transformation is that it renders convolutions to products. In fact, denoting the moments of $P_{a b}^{T}$ and $D_{a}^{h}$ by $A_{a b}$ and $M_{a}$, respectively, we have

$$
\begin{aligned}
& \frac{d}{d \ln Q^{2}} M_{i}^{-}\left(n, Q^{2}\right)=\frac{\alpha_{S}\left(Q^{2}\right)}{2 \pi} A_{N S}\left(n, \alpha_{S}\left(Q^{2}\right)\right) M_{i}^{-}\left(n, Q^{2}\right), \\
& \frac{d}{d \ln Q^{2}} M_{i}^{+}\left(n, Q^{2}\right)=\frac{\alpha_{S}\left(Q^{2}\right)}{2 \pi} A_{N S}\left(n, \alpha_{S}\left(Q^{2}\right)\right) M_{i}^{+}\left(n, Q^{2}\right), \\
& \frac{d}{d \ln Q^{2}} M_{\Sigma}\left(n, Q^{2}\right)=\frac{\alpha_{S}\left(Q^{2}\right)}{2 \pi}\left(A_{q q}\left(n, \alpha_{S}\left(Q^{2}\right)\right) M_{\Sigma}\left(n, Q^{2}\right)+A_{g q}\left(n, \alpha_{S}\left(Q^{2}\right)\right) M_{G}\left(n, Q^{2}\right)\right), \\
& \frac{d}{d \ln Q^{2}} M_{G}\left(n, Q^{2}\right)=\frac{\alpha_{S}\left(Q^{2}\right)}{2 \pi}\left(A_{q g}\left(n, \alpha_{S}\left(Q^{2}\right)\right) M_{\Sigma}\left(n, Q^{2}\right)+A_{g g}\left(n, \alpha_{S}\left(Q^{2}\right)\right) M_{G}\left(n, Q^{2}\right)\right),
\end{aligned}
$$


where $A_{N S}$ refers to the usual non-singlet combination of quark and antiquark splitting functions [12, and

$$
\begin{aligned}
M_{i}^{-}\left(n, Q^{2}\right) & =\frac{1}{2}\left(M_{q_{i}}\left(n, Q^{2}\right)-M_{\bar{q}_{i}}\left(n, Q^{2}\right)\right) \\
M_{i}^{+}\left(n, Q^{2}\right) & =\frac{1}{2}\left(M_{q_{i}}\left(n, Q^{2}\right)+M_{\bar{q}_{i}}\left(n, Q^{2}\right)\right)-\frac{1}{2 N_{f}} M_{\Sigma}\left(n, Q^{2}\right) \\
M_{\Sigma}\left(n, Q^{2}\right) & =\sum_{i=1}^{N_{f}}\left(M_{q_{i}}\left(n, Q^{2}\right)+M_{\bar{q}_{i}}\left(n, Q^{2}\right)\right) \\
M_{G}\left(n, Q^{2}\right) & =M_{g}\left(n, Q^{2}\right) .
\end{aligned}
$$

The LO expressions for $A_{a b}$ may be found in [12,13] and the NLO expressions in [14] $]^{2}$. Note that the non-singlet terms, $M_{i}^{ \pm}$, decouple from the gluon. The solutions of (11) factorize,

$$
M\left(n, Q^{2}\right)=E\left(Q_{0}^{2}, Q^{2}\right) M\left(n, Q_{0}^{2}\right)
$$

The relevant evolution operators, $E\left(Q_{0}^{2}, Q^{2}\right)$, may be found in 12]. We parameterize the $x$ dependence of the fragmentation functions at $Q_{0}^{2}$ as

$$
D\left(x, Q_{0}^{2}\right)=N x^{\alpha}(1-x)^{\beta}
$$

The corresponding moments read

$$
M\left(n, Q_{0}^{2}\right)=N B(n+\alpha, \beta+1)
$$

where $B$ is Euler's beta function. The evolution in $n$ space is performed analytically via (13). Finally, the $D\left(x, Q^{2}\right)$ functions are obtained by solving (10) numerically. This requires knowledge of $A_{a b}$ for complex $n$. A prescription for the proper analytic continuation may be found in [15].

In (14), we introduced three parameters for each parton. To reduce the number of free parameters, we shall discriminate only between valence- and sea-type quarks. To that

\footnotetext{
${ }^{1}$ Note that there is a typographical error in (B.46c) of [13]. $C_{F}$ should be replaced by $C_{A}$.

${ }^{2}$ Note that there is a typographical error in (A.10) of [14]. The term before the last one of the contribution proportional to $C_{F} T_{R} f$ should read $-\left(16 / 3(n+2)^{2}\right)$ instead of $-\left(16 / 3(n+1)^{2}\right)$.
} 
end, we make the following ansatz for the average of charged pions,

$$
\begin{aligned}
D_{V}^{\pi}+D_{S}^{\pi} & =D_{u}^{\pi}=D_{\bar{u}}^{\pi}=D_{d}^{\pi}=D_{\bar{d}}^{\pi} \\
D_{S}^{\pi} & =D_{s}^{\pi}=D_{\bar{s}}^{\pi}=D_{c}^{\pi}=D_{\bar{c}}^{\pi}=D_{b}^{\pi}=D_{\bar{b}}^{\pi} .
\end{aligned}
$$

For the average of the charged kaons, we set

$$
\begin{aligned}
D_{V}^{K}+D_{S}^{K} & =D_{u}^{K}=D_{\bar{u}}^{K}=D_{s}^{K}=D_{\bar{s}}^{K} \\
D_{S}^{K} & =D_{d}^{K}=D_{\bar{d}}^{K}=D_{c}^{K}=D_{\bar{c}}^{K}=D_{b}^{K}=D_{\bar{b}}^{K} .
\end{aligned}
$$

Furthermore, we put $\alpha=-1$ for valence and sea quarks, which may be motivated by inspection of the energy dependence of hadronic multiplicities. In the case of the gluon, we found that the choice $\alpha=0$ is favoured by the data. We are thus left with six parameters to be determined from experiment.

\section{Results}

In general, data of inclusive hadron production at $e^{+} e^{-}$colliders are most suitable for the extraction of fragmentation functions; in the case of fixed-target, collider, and $e p$ data, the information on the fragmentation functions is obscured by theoretical uncertainties arising from the parton density functions and the choice of factorization scales connected with the initial state. For our analysis, we select the data on charged-pion and -kaon production taken at energy $\sqrt{s}=29 \mathrm{GeV}$ by the TPC Collaboration at SLAC 16]. These data combine small statistical errors with fine binning in $x$ and are thus more constraining than data collected by other $e^{+} e^{-}$experiments in the energy range from 5.2 to $44 \mathrm{GeV}$. For this reason, our approach is to fit exclusively to TPC data and to use the other data for cross checks.

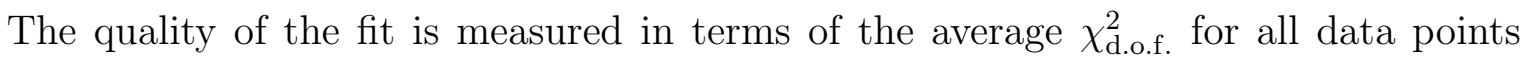
with $x>x_{\text {cut }}=2 \mathrm{GeV} / \sqrt{s}$. This restriction is necessary to exclude events in the nonperturbative region, where the above formalism is bound to fail. Our technical procedure is as follows. We consider (田) at $\mu=M_{f}=\sqrt{s}=29 \mathrm{GeV}$ as a function of the six parameters 
that determine the $x$ dependence of the fragmentation functions at $Q_{0}^{2}=2 \mathrm{GeV}^{2}$. Using a multidimensional minimization algorithm [17], we search this six-dimensional parameter space for the point at which the deviation of the theoretical prediction from the data becomes minimal. Our results are listed below. For the average of charged pions, we obtain

$$
\begin{aligned}
& D_{V}^{(\pi, L O)}\left(x, Q_{0}\right)=0.551 x^{-1}(1-x)^{1.2} \\
& D_{S}^{(\pi, L O)}\left(x, Q_{0}\right)=1.23 x^{-1}(1-x)^{4.77} \\
& D_{G}^{(\pi, L O)}\left(x, Q_{0}\right)=3.77(1-x)^{2.0}
\end{aligned}
$$

in $\mathrm{LO}$ and

$$
\begin{aligned}
& D_{V}^{(\pi, N L O)}\left(x, Q_{0}\right)=0.338 x^{-1}(1-x)^{1.2}, \\
& D_{S}^{(\pi, N L O)}\left(x, Q_{0}\right)=1.19 x^{-1}(1-x)^{5.26}, \\
& D_{G}^{(\pi, N L O)}\left(x, Q_{0}\right)=3.65(1-x)^{2.0}
\end{aligned}
$$

in NLO. In the case of kaons, we find

$$
\begin{aligned}
& D_{V}^{(K, L O)}\left(x, Q_{0}\right)=0.155 x^{-1}(1-x)^{1.4}, \\
& D_{S}^{(K, L O)}\left(x, Q_{0}\right)=0.216 x^{-1}(1-x)^{1.69}, \\
& D_{G}^{(K, L O)}\left(x, Q_{0}\right)=0.324(1-x)^{2.0}
\end{aligned}
$$

in $\mathrm{LO}$ and

$$
\begin{aligned}
& D_{V}^{(K, N L O)}\left(x, Q_{0}\right)=0.099 x^{-1}(1-x)^{1.4} \\
& D_{S}^{(K, N L O)}\left(x, Q_{0}\right)=0.214 x^{-1}(1-x)^{2.16} \\
& D_{G}^{(K, N L O)}\left(x, Q_{0}\right)=0.372(1-x)^{2.0}
\end{aligned}
$$

in NLO. For the reader's convenience, we list simple parameterizations of the $x$ and $Q^{2}$ dependences of these sets in the Appendix. It cannot be expected that such simple parameterizations reproduce the true result perfectly for all $x$ and $Q^{2}$ values that occur in applications. Deviations in excess of $10 \%$ may occur at $Q^{2}<25 \mathrm{GeV}^{2}$ and $x<0.1$, 
in particular for the gluon. Nevertheless, we believe that such parameterizations are indispensable for practical purposes, especially at NLO.

Our fragmentation functions exhibit good perturbative stability. In the case of the pions, there is only little difference between our LO and NLO sets. The difference is somewhat bigger for the kaons. In the comparisons with data, the NLO sets do just slightly better. The $x$ dependence of (19.21) at $Q_{0}^{2}$ is visualized in Figs. 1.2, respectively. In the following, we shall always employ the parameterizations given in the Appendix. The goodness of our fits to the TPC data on charged-pion and -kaon production may be judged from Figs. 目, respectively. In LO (NLO), we achieve $\chi_{\text {d.o.f. }}^{2}=0.7(0.7)$ for pions and $0.6(0.5)$ for kaons. Here and in the following, $\chi_{\text {d.o.f. }}^{2}$ refers to the interval $\max \left(x_{\text {cut }}, 0.1\right) \leq x \leq 0.9$. Notice that our parameterizations fit the TPC data excellently even down to $x=0.06$.

In Figs. 107, we postdict data taken by DASP [18] and ARGUS [19] at $\sqrt{s}=5.2$ and $10 \mathrm{GeV}$, respectively. We have made comparisons also with TASSO data at $\sqrt{s}=34$ and $44 \mathrm{GeV}$ [20], which we do not, however, display graphically. Data of inclusive chargedkaon production are well reproduced by our fits. In Fig. 0, this is nicely demonstrated for ARGUS. In the case of the charged-kaon data by DASP, for which we do not show a figure, we reach $\chi_{\text {d.o.f. }}^{2}=1.4(1.2)$ in $\mathrm{LO}(\mathrm{NLO})$, which we can bring down to values below unity when we exploit the freedom in the overall normalization of the data by about $\pm 12 \%$.

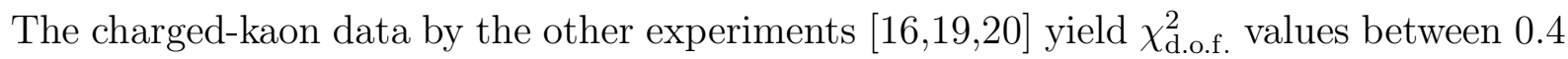
and 0.6 without adjustment, both in LO and NLO.

The situation is slightly less favourable in the case of charged pions. The pion data by DASP [18] behave similarly as their kaon data (see Fig. 5). However, the ARGUS data [19] are inconsistent intrinsically at large $x$, which leads to increased $\chi_{\text {d.o.f. }}^{2}$ values, 2.2 in LO and 1.8 in NLO (see Fig. (6). For $x<0.1$, the theoretical curves overshoot the data. This is presumably attributed to mass effects, which have been ignored in our approach. As per construction, the TPC data are reproduced well, with $\chi_{\text {d.o.f. }}^{2}=0.7$ both in LO

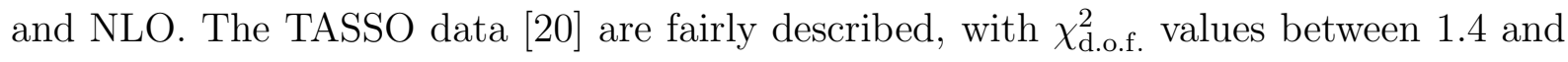
2.0. However, these data are of limited use for our purposes because, for $x>0.2$, their 
binning is a factor of 3 to 5 coarser than that of the TPC data. Furthermore, the data sets at $\sqrt{s}=34$ and $44 \mathrm{GeV}$ do not comply with the expectation that scaling violation renders spectra at higher energies steeper. Leaving aside the TASSO data, the goodness of our charged-pion fits is quite satisfactory.

\section{Applications}

Since fragmentation functions are assumed to be universal, i.e., independent of the process from which they have been extracted, we can apply them to make predictions for inclusive particle production at fixed-target, collider, and $e p$ scattering experiments, too. In the following, we shall confine ourselves to more extended studies of $e^{+} e^{-}$annihilation, which will substantiate our confidence in the virtue of our sets. Work on inclusive particle production in NLO at HERA and $p \bar{p}$ colliders is in progress.

We commence by elaborating a welcome generalization of our results. By SU(2) symmetry, our charged-pion sets are valid also for the neutrals. This observation is supported by the inspection of neutral-pion data, e.g., by ARGUS [21], TPC [22], JADE [23], and CELLO [24, which agree with the respective charged-pion data within the errors quoted. This is reflected also by the $\chi_{\text {d.o.f. }}^{2}$ analysis. Cross checks with new data by L3 25] prove to be very successful, too. By analogous theoretical considerations, we can establish a relationship between the fragmentation functions for the average of the $K^{0}$ and $\bar{K}^{0}$ mesons and those for the charged kaons. Specifically, the $u, \bar{u}$ and $d, \bar{d}$ fragmentation functions swop places in (17). Again, we find reasonable agreement way up to LEP 1 energy [19,25,26,27,28].

As a second application, we deal with inclusive charged-hadron production. I.e., we consider experiments that observe charged tracks without identifying the particle species. In this context, it has become customary to sum over the produced hadrons rather than to form the average. Clearly, pions and kaons make up the bulk of the cross section. By including also protons and antiprotons, the next important subset, we obtain a good approximation for the sum of all charged hadrons. Inspection of TPC data [16] reveals 
that the averaged cross section of inclusive production of protons or antiprotons has a shape similar to the one of charged pions, but is reduced in size by a factor of $0.16 \pm 0.02$. Thus, we take the hadronic cross section to be

$$
\frac{d \sigma^{\left(h^{+}+h^{-}\right)}}{d x}=2\left[(1+0.16) \frac{d \sigma^{\left(\pi^{+}+\pi^{-}\right) / 2}}{d x}+\frac{d \sigma^{\left(K^{+}+K^{-}\right) / 2}}{d x}\right] .
$$

We compare this prediction with high-precision data by DELPHI [29] in Fig. 8 and find good agreement, with $\chi_{\text {d.o.f. }}^{2}=0.5(1.0)$ in LO (NLO). Our results also agree well with data by MARK II [30], AMY [31], TASSO [32], and CELLO [33]. Whereas the $x$ dependence is reproduced perfectly for the MARK II, AMY, and DELPHI data, there are minor deviations in the case of TASSO and CELLO. Except for DELPHI, the $\chi_{\text {d.o.f. values }}^{2}$ exceed unity in all these cases, which indicates that the incorporation of baryons by an overall $Q^{2}$-independent factor is perhaps to naïve.

In order to test scaling violation, we compute $(22)$ at $\sqrt{s}=91$ and $29 \mathrm{GeV}$, plot the ratio as a function of $x$ in Fig. 9, and compare the answer with the corresponding ratio of DELPHI [29] and MARK II data [30], taking into account only the statistical errors of the latter. For $x>0.1$, our prediction agrees remarkably well with the experimental result, both in LO (dashed line) and NLO (solid line). We note in passing that a similar analysis was carried out recently by Nason and Webber [8], who encountered a certain mismatch between their calculation and their choice of data. These authors determined a set of fragmentation functions by fitting to the DELPHI data on inclusive charged-hadron production [29], evolved their results backwards down to PETRA energy, and compared the outcome with TASSO data [32], taking into account only the statistical errors of the latter. They interpreted the discrepancy that they discovered by suggesting that significant nonperturbative power corrections are necessary to reproduce the measured scaling violation (see Fig. 14 of [8]). In our opinion, the major source of this problem is the neglect of the systematic errors on the TASSO data rather than the influence of power corrections. In fact, when we repeat the analysis of Fig. 9 for TASSO and CELLO [33] data, both taken at PETRA, again including only the statistical errors in the denominator, we find that the two sets do not overlap and are separated by our curves. This suggests 
that the TASSO and CELLO data are not consistent with each other when only their statistical errors are taken into account.

Another look at scaling violation is presented in Fig. 10, which shows the $Q^{2}$ dependence of the charged-hadron cross section for various values of $x$. The evaluations of (22) to LO (dashed lines) and NLO (solid lines) are confronted with a rich collection of data 18, $19,29,30,31,32,33$, which have been interpolated in $x$ where appropriate. We conclude that the data indeed exhibit the expected scaling violation.

The DELPHI Collaboration used an alternative approach to interpret their data [29]. The inclusive hadron spectra are generated from a Monte Carlo program based on order $\alpha_{S}^{2}$ QCD matrix elements, which produces jet cross sections and takes into account the subsequent fragmentation of the jets into hadrons according to certain model assumptions. In this approach, the cancellation of infrared and collinear singularities occurs through the definition of jet cross sections with chosen jet resolution criteria. This deviates from the canonical factorization approach, where the collinear singularities are absorbed into the fragmentation functions. We strongly advocate the canonical formalism, which is intrinsic to the QCD-improved parton model.

At this point, we should address a possible shortcoming of our analysis, which is common to all studies of fragmentation in $e^{+} e^{-}$annihilation, namely the circumstance that the gluon fragmentation function is poorly determined. This is due to the fact that the gluon participates in the process only at NLO, and even then has an appreciable impact on the cross section only at very small $x$ values close to $x_{\text {cut }}$. Thus, it contributes mainly through the $Q^{2}$ evolution, where it couples to the singlet combination of quarks. Consequently, the gluon parameters are strongly correlated with the sea-quark parameters and are feebly constrained by the data. Hadron and ep scattering experiments deliver far more information on the gluon fragmentation function. Fortunately, the new three-jet data by OPAL [34] are able to probe gluon fragmentation directly. In Fig. 11], we compare these data with our LO (dashed line) and NLO predictions (solid line) according to (22). The agreement is reasonable. We tried also different ansätze for $D_{G}\left(x, Q_{0}^{2}\right)$ by choosing $\alpha \neq 0$ in (14). While they worked similarly well for all $e^{+} e^{-}$data considered above, they 
TABLE I. Left-hand side of (23) at NLO for $\sqrt{Q^{2}}=5$, 29, and $91 \mathrm{GeV}$. We use $\min \left(x_{\text {cut }}, 0.1\right)$ as the lower bound of integration and sum over pions, kaons, protons, and antiprotons.

\begin{tabular}{|c|c|c|c|}
\hline \multirow{2}{*}{$a$} & \multicolumn{3}{|c|}{$\sqrt{Q^{2}}[\mathrm{GeV}]$} \\
\cline { 2 - 4 } & 5 & 29 & 91 \\
\hline$u, d$ & 0.81 & 0.76 & 0.94 \\
$s$ & 0.55 & 0.52 & 0.68 \\
$c, b$ & 0.45 & 0.45 & 0.60 \\
$g$ & 0.62 & 0.58 & 0.83 \\
\hline
\end{tabular}

failed in the case of Fig. 11 [35].

Finally, we investigate if our fragmentation functions satisfy the momentum sum rules. Guided by the idea that a given outgoing parton, $a$, will fragment with $100 \%$ likelihood into some hadron, $h$, and that momentum is conserved during the fragmentation process, we expect that

$$
\sum_{h} \int_{0}^{1} d x x D_{a}^{h}\left(x, Q^{2}\right)=1
$$

holds for any value of $Q^{2}$. In our analysis, the left-hand-side of (23) should be smaller than unity, since we consider only pions, kaons, protons, and antiprotons, while we do not include hyperon channels. Furthermore, we are forced to introduce a finite lower bound of integration in (23), since the $x \rightarrow 0$ limit of $D_{a}^{h}\left(x, Q^{2}\right)$ is beyond our control. $x_{\min }$ is a plausible choice for this cutoff, except at small $Q^{2}\left(\sqrt{Q^{2}}<20 \mathrm{GeV}\right)$, where we use 0.1 instead. In Table I, we list the results obtained with our NLO fragmentation functions for $\sqrt{Q^{2}}=5,29$, and $91 \mathrm{GeV}$. In fact, all entries are smaller than one as they should.

\section{Conclusions and Outlook}

We presented LO and NLO fragmentation functions for charged pions and kaons in the $\overline{\mathrm{MS}}$ scheme. They were extracted from $e^{+} e^{-}$data on inclusive production of these particles collected at $\sqrt{s}=29 \mathrm{GeV}$. We checked that these fragmentation functions are in agree- 
ment with the majority of the $e^{+} e^{-}$data in the energy range between $5.2 \mathrm{GeV}$ and $M_{Z}$, and suggested possible explanations, where this was not the case. This work is motivated by the circumstance that a great amount of high-quality data on inclusive hadron production has been accumulated in fixed-target, hadron collider, ep scattering, and $e^{+} e^{-}$annihilation experiments since the early 1980's, which was not accompanied by any progress in the phenomenology of charged-particle fragmentation functions. The quest for such progress has been increased dramatically by the advent of new high-statistics data from HERA and LEP 1. In particular, meaningful tests of the QCD-improved parton model can be performed only beyond LO, so that knowledge of fragmentation functions with NLO evolution is essential. The present paper makes an effort to fill this gap.

By the factorization theorem, the use of our fragmentation functions is not restricted to $e^{+} e^{-}$collisions. They may be applied to any other process by which charged pions and kaons are produced inclusively. However, one should bear in mind that our sets come with a certain range of validity, which is specified in the Appendix. For instance, the production of low- $p_{T}$ hadrons in high-energy hadron-hadron and $e p$ scattering has a tendency to probe small values of $x$ that lie outside this range. Moreover, such hadrons are likely to originate from gluons, whose fragmentation function is not yet constrained so well by $e^{+} e^{-}$data. This leaves room for further improvements in the future.

\section{ACKNOWLEDGMENTS}

We thank J.Ph. Guillet for clarifying comments regarding [6].

\section{A Parameterizations}

For the reader's convenience, we present here simple parameterizations of the $x$ and $Q^{2}$ dependence of our fragmentation functions. It is necessary to quote a range of validity for these parameterizations. In the case of $Q^{2}$, they agree with the results obtained by explicit evolution to better than $10 \%$ if $5 \mathrm{GeV} \leq \sqrt{Q^{2}} \leq 91 \mathrm{GeV}$. As for $x$, we are experiencing technical limitations connected with the stability of the numerical integration for $x \rightarrow 0$ 
and $x \rightarrow 1$. Our results should be reliable in the interval $0.1 \leq x \leq 0.9$. The upper bound on $x$ may be ignored in practice, since the fragmentation functions take very small values at $x=0.9$ and approach zero for $x \rightarrow 1$.

As usual, we introduce the scaling variable

$$
\bar{s}=\ln \frac{\ln \left(Q^{2} / \Lambda^{2}\right)}{\ln \left(Q_{0}^{2} / \Lambda^{2}\right)}
$$

and parameterize our results by simple functions in $x$ with coefficients that are polynomials in $\bar{s}$. We find that the template

$$
D\left(x, Q^{2}\right)=N x^{\alpha}(1-x)^{\beta}(1+x)^{\gamma}
$$

works best in our case. We now list the parameters to be inserted in (25) for charged pions and kaons both in LO and NLO. At $Q^{2}=Q_{0}^{2}$, these parameterizations coincide with (18 21) as they should.

1. LO fragmentation functions for $\left(\pi^{+}+\pi^{-}\right) / 2$ :

- $D_{V}^{(\pi, L O)}\left(x, Q^{2}\right)$ :

$$
\begin{aligned}
N & =0.551-0.053 \bar{s}-0.032 \bar{s}^{2} \\
\alpha & =-1.0-0.0272 \bar{s} \\
\beta & =1.2+0.67 \bar{s} \\
\gamma & =-0.393 \bar{s}
\end{aligned}
$$

- $D_{S}^{(\pi, L O)}\left(x, Q^{2}\right)$ :

$$
\begin{aligned}
& N=1.23+2.85 \bar{s}-1.60 \bar{s}^{2} \\
& \alpha=-1.0+0.447 \bar{s}-0.266 \bar{s}^{2} \\
& \beta=4.77-2.88 \bar{s}+2.05 \bar{s}^{2} \\
& \gamma=-9.01 \bar{s}+4.36 \bar{s}^{2}
\end{aligned}
$$


- $D_{G}^{(\pi, L O)}\left(x, Q^{2}\right)$ :

$$
\begin{aligned}
N & =3.77-5.49 \bar{s}+2.14 \bar{s}^{2} \\
\alpha & =-1.73 \bar{s}+0.329 \bar{s}^{2} \\
\beta & =2.0+1.00 \bar{s}+0.44 \bar{s}^{2} \\
\gamma & =-2.05 \bar{s}+2.48 \bar{s}^{2}
\end{aligned}
$$

2. NLO fragmentation functions for $\left(\pi^{+}+\pi^{-}\right) / 2$ :

- $D_{V}^{(\pi, N L O)}\left(x, Q^{2}\right)$ :

$$
\begin{aligned}
N & =0.338-0.064 \bar{s}-0.0105 \bar{s}^{2} \\
\alpha & =-1.0-0.059 \bar{s} \\
\beta & =1.2+0.60 \bar{s} \\
\gamma & =-0.163 \bar{s}
\end{aligned}
$$

- $D_{S}^{(\pi, N L O)}\left(x, Q^{2}\right)$ :

$$
\begin{aligned}
& N=1.19+4.20 \bar{s}-2.86 \bar{s}^{2} \\
& \alpha=-1.0+0.757 \bar{s}-0.537 \bar{s}^{2} \\
& \beta=5.26-5.22 \bar{s}+3.62 \bar{s}^{2} \\
& \gamma=-13.6 \bar{s}+8.17 \bar{s}^{2}
\end{aligned}
$$

- $D_{G}^{(\pi, N L O)}\left(x, Q^{2}\right)$ :

$$
\begin{aligned}
& N=3.65-6.00 \bar{s}+2.52 \bar{s}^{2} \\
& \alpha=-1.49 \bar{s}-0.213 \bar{s}^{2} \\
& \beta=2.0-0.23 \bar{s}+1.52 \bar{s}^{2} \\
& \gamma=-6.53 \bar{s}+8.43 \bar{s}^{2}
\end{aligned}
$$

3. LO fragmentation functions for $\left(K^{+}+K^{-}\right) / 2$ : 
- $D_{V}^{(K, L O)}\left(x, Q^{2}\right)$ :

$$
\begin{aligned}
N & =0.155-0.010 \bar{s}-0.010 \bar{s}^{2} \\
\alpha & =-1.0-0.012 \bar{s} \\
\beta & =1.4+0.66 \bar{s} \\
\gamma & =-0.572 \bar{s}
\end{aligned}
$$

- $D_{S}^{(K, L O)}\left(x, Q^{2}\right)$ :

$$
\begin{aligned}
N & =0.216-0.0744 \bar{s} \\
\alpha & =-1.0-0.196 \bar{s} \\
\beta & =1.69+0.69 \bar{s} \\
\gamma & =-0.195 \bar{s}
\end{aligned}
$$

- $D_{G}^{(K, L O)}\left(x, Q^{2}\right)$ :

$$
\begin{aligned}
N & =0.324-0.506 \bar{s}+0.205 \bar{s}^{2} \\
\alpha & =-1.860 \bar{s} \\
\beta & =2.0+1.67 \bar{s} \\
\gamma & =-0.235 \bar{s}+2.68 \bar{s}^{2}
\end{aligned}
$$

4. NLO fragmentation functions for $\left(K^{+}+K^{-}\right) / 2$ :

- $D_{V}^{(K, N L O)}\left(x, Q^{2}\right)$ :

$$
\begin{aligned}
N & =0.099-0.0198 \bar{s}-0.0027 \bar{s}^{2} \\
\alpha & =-1.0-0.060 \bar{s} \\
\beta & =1.4+0.60 \bar{s} \\
\gamma & =-0.233 \bar{s}
\end{aligned}
$$

- $D_{S}^{(K, N L O)}\left(x, Q^{2}\right)$ :

$$
N=0.214-0.00073 \bar{s}-0.0063 \bar{s}^{2}
$$




$$
\begin{aligned}
& \alpha=-1.0-0.0067 \bar{s} \\
& \beta=2.16+0.57 \bar{s} \\
& \gamma=-0.912 \bar{s}
\end{aligned}
$$

- $D_{G}^{(K, N L O)}\left(x, Q^{2}\right)$ :

$$
\begin{aligned}
& N=0.372-0.606 \bar{s}+0.253 \bar{s}^{2} \\
& \alpha=-2.55 \bar{s}+0.584 \bar{s}^{2} \\
& \beta=2.0-0.52 \bar{s}+1.82 \bar{s}^{2} \\
& \gamma=-7.43 \bar{s}+9.24 \bar{s}^{2}
\end{aligned}
$$

\section{References}

[1] P. Mättig: Phys. Rep. 177 (1989) 141

[2] F.M. Borzumati, B.A. Kniehl, G. Kramer: Z. Phys. C57 (1993) 595

[3] F.M. Borzumati, B.A. Kniehl, G. Kramer: Z. Phys. C59 (1993) 341; B.A. Kniehl, G. Kramer: Z. Phys. C62 (1994) 53

[4] R. Baier, J. Engels, B. Petersson: Z. Phys. C2 (1979) 265

[5] M. Anselmino, P. Kroll, E. Leader: Z. Phys. C18 (1983) 307

[6] P. Chiappetta, M. Greco, J.-Ph. Guillet, S. Rolli, M. Werlen: Nucl. Phys. B412 (1994) 3

[7] M. Greco, S. Rolli: Z. Phys. C60 (1993) 169; M. Greco, S. Rolli, A. Vicini: Preprint DFPD 94/TH/21 and FERMILAB-Pub-94/075-A

[8] P. Nason, B.R. Webber: Nucl. Phys. B421 (1994) 473

[9] G. Altarelli, R.K. Ellis, G. Martinelli, S.Y. Pi: Nucl. Phys. B160 (1979) 301; R. Baier, K. Fey: Z. Phys. C2 (1979) 339 
[10] K. Hikasa et al. (Particle Data Group): Phys. Rev. D45 (1992) S1

[11] V.N. Gribov, L.N. Lipatov: Yad. Fiz. 15 (1972) 781 [Sov. J. Nucl. Phys. 15 (1972) 438]; G. Altarelli, G. Parisi: Nucl. Phys. B126 (1977) 298; Yu.L. Dokshitser: Zh. Eksp. Teor. Fiz. 73 (1977) 1216 [Sov. Phys. JETP 46 (1977) 641]

[12] W. Furmanski, R. Petronzio: Z. Phys. C11 (1982) 293; G. Curci, W. Furmanski, R. Petronzio: Nucl. Phys. B175 (1980) 27; W. Furmanski, R. Petronzio: Phys. Lett. 97B (1980) 437

[13] E.G. Floratos, C. Kounnas, R. Lacaze: Nucl. Phys. B192 (1981) 417

[14] M. Glück, E. Reya, A. Vogt: Phys. Rev. D48 (1993) 116

[15] M. Glück, E. Reya, A. Vogt: Phys. Rev. D45 (1992) 3986

[16] H. Aihara et al., TPC Coll.: Phys. Rev. Lett. 61 (1988) 1263; Preprint LBL-23737 (1988)

[17] MINUIT, CERN Fortran Library (1989)

[18] R. Brandelik et al., DASP Coll.: Nucl. Phys. B148 (1979) 189

[19] H. Albrecht et al., ARGUS Coll.: Z. Phys. C44 (1989) 547; Z. Phys. C54 (1992) 13

[20] W. Braunschweig et al., TASSO Coll.: Z. Phys. C42 (1989) 189

[21] H. Albrecht et al., ARGUS Coll.: Z. Phys. C46 (1990) 15

[22] H. Aihara et al., TPC Coll.: Z. Phys. C27 (1985) 187

[23] D.D. Pitzl et al., JADE Coll.: Z. Phys. C46 (1990) 1

[24] H.J. Behrend et al., CELLO Coll.: Z. Phys. C47 (1990) 1

[25] M. Acciarri et al., L3 Coll.: Preprint CERN-PPE/94-53 (March 1994)

[26] S. Abachi et al., HRS Coll.: Phys. Rev. D41 (1990) 2045 
[27] W. Braunschweig et al., TASSO Coll.: Z. Phys. C47 (1990) 167

[28] D. Buskulic, ALEPH Coll.: Preprint CERN-PPE/94-74 (May 1994)

[29] P. Abreu et al., DELPHI Coll.: Phys. Lett. B311 (1993) 408

[30] A. Peterson et al., MARK II Coll.: Phys. Rev. D37 (1988) 1

[31] Y.K. Li et al., AMY Coll.: Phys. Rev. D41 (1990) 2675; T. Kumita et al., AMY Coll.: Phys. Rev. D42 (1990) 1339

[32] W. Braunschweig et al., TASSO Coll.: Z. Phys. C47 (1990) 187

[33] H.J. Behrend et al., CELLO Coll.: to be published. Data were extracted from [29]

[34] P.D. Acton et al., OPAL Coll.: Z. Phys. C58 (1993) 387. Data were extracted from 8)

[35] J. Binnewies: Diploma thesis (May 1994) 


\section{FIGURE CAPTIONS}

Figure 1: $x$ distributions of the NLO pion fragmentation functions at $Q_{0}^{2}=2 \mathrm{GeV}^{2}$ Figure 2: $x$ distributions of the NLO kaon fragmentation functions at $Q_{0}^{2}=2 \mathrm{GeV}^{2}$ Figure 3: Cross section of inclusive charged-pion production at $\sqrt{s}=29 \mathrm{GeV}$ as a function of $x$. The LO (dashed line) and NLO fits (solid line) are compared with TPC data 16] Figure 4: Cross section of inclusive charged-kaon production at $\sqrt{s}=29 \mathrm{GeV}$ as a function of $x$. The LO (dashed line) and NLO fits (solid line) are compared with TPC data 16 Figure 5: Same as in Fig. 司 for $\sqrt{s}=5.2 \mathrm{GeV}$ and DASP data [18]

Figure 6: Same as in Fig. 通 for $\sqrt{s}=10 \mathrm{GeV}$ and ARGUS data 19

Figure 7: Same as in Fig. [- for $\sqrt{s}=10 \mathrm{GeV}$ and ARGUS data [19]

Figure 8: Cross section of inclusive charged-hadron production at $\sqrt{s}=91 \mathrm{GeV}$ as a function of $x$. The dashed (solid) line corresponds to the prediction on the basis of (22) at LO (NLO)

Figure 9: Ratio of the $x$ distributions of inclusive charged-hadron production at $\sqrt{s}=91$ and $29 \mathrm{GeV}$. The dashed (solid) line corresponds to the prediction on the basis of (22) at LO (NLO). The points correspond to DELPHI data [29] divided by MARK II data [30], taking into account only the statistical errors of the latter

Figure 10: Scaling violation in the cross section of inclusive charged-hadron production. Shown is the $Q^{2}$ dependence for $x=0.075,0.15,0.25,0.35,0.5,0.75,0.9$. The dashed (solid) line corresponds to the prediction on the basis of (22) to LO (NLO). The data are taken from [18, [9, 29, 30,31,32, 33] and are partly interpolated in $x$

Figure 11: Cross section of inclusive charged-hadron production at $\sqrt{s}=91 \mathrm{GeV}$ as a function of $x$. The dashed (solid) line corresponds to the prediction on the basis of (22) at LO (NLO). The data are taken by OPAL [34] and represent the first direct measurement of the gluon fragmentation function for charged hadrons 
This figure "fig1-1.png" is available in "png" format from: http://arxiv.org/ps/hep-ph/9407347v2 
This figure "fig1-2.png" is available in "png" format from: http://arxiv.org/ps/hep-ph/9407347v2 
This figure "fig1-3.png" is available in "png" format from: http://arxiv.org/ps/hep-ph/9407347v2 
This figure "fig1-4.png" is available in "png" format from: http://arxiv.org/ps/hep-ph/9407347v2 
This figure "fig1-5.png" is available in "png" format from: http://arxiv.org/ps/hep-ph/9407347v2 
This figure "fig1-6.png" is available in "png" format from: http://arxiv.org/ps/hep-ph/9407347v2 
This figure "fig1-7.png" is available in "png" format from: http://arxiv.org/ps/hep-ph/9407347v2 
This figure "fig1-8.png" is available in "png" format from: http://arxiv.org/ps/hep-ph/9407347v2 
This figure "fig1-9.png" is available in "png" format from: http://arxiv.org/ps/hep-ph/9407347v2 
This figure "fig1-10.png" is available in "png" format from: http://arxiv.org/ps/hep-ph/9407347v2 
This figure "fig1-11.png" is available in "png" format from: http://arxiv.org/ps/hep-ph/9407347v2 


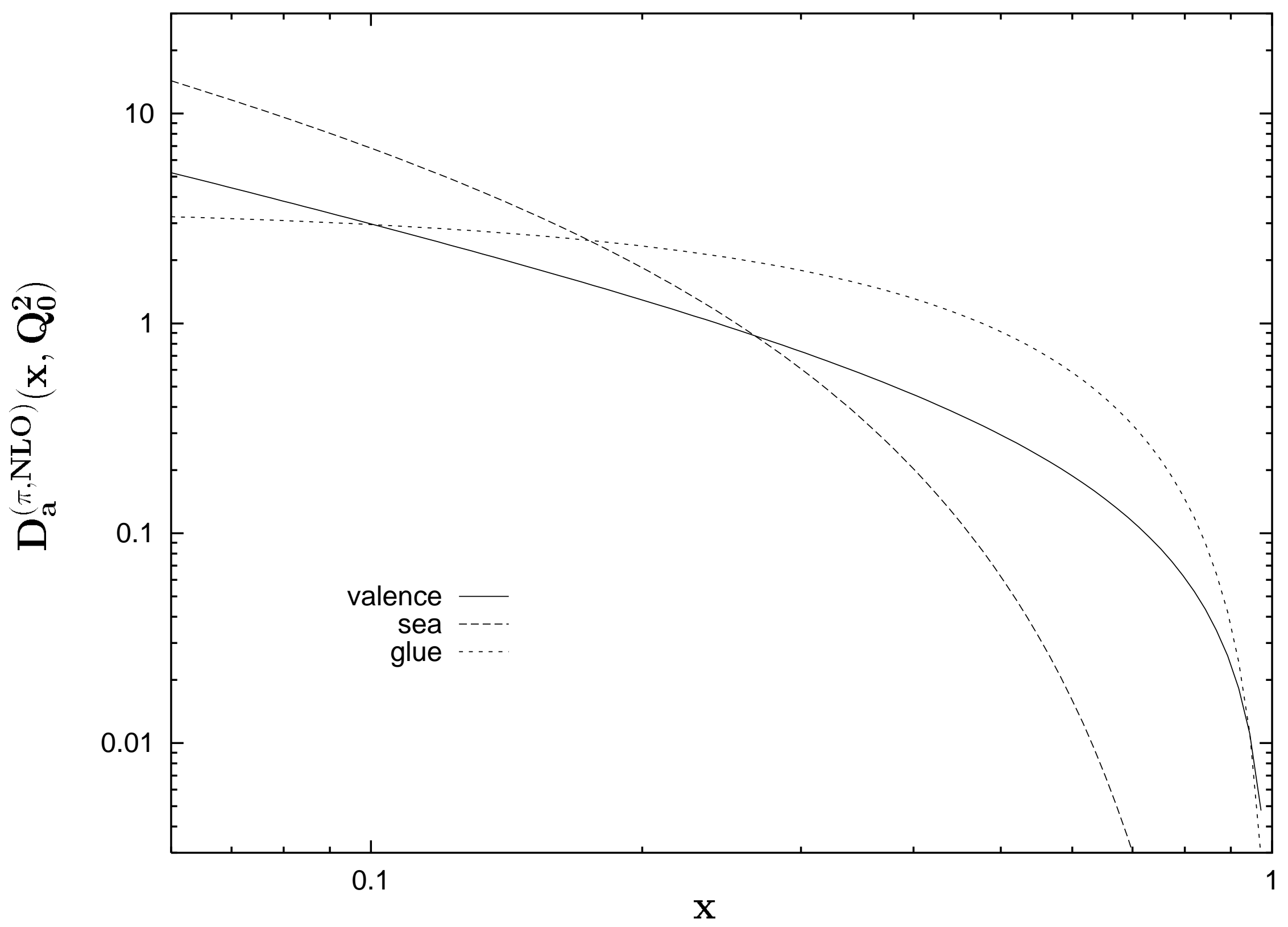

Fig. 1 


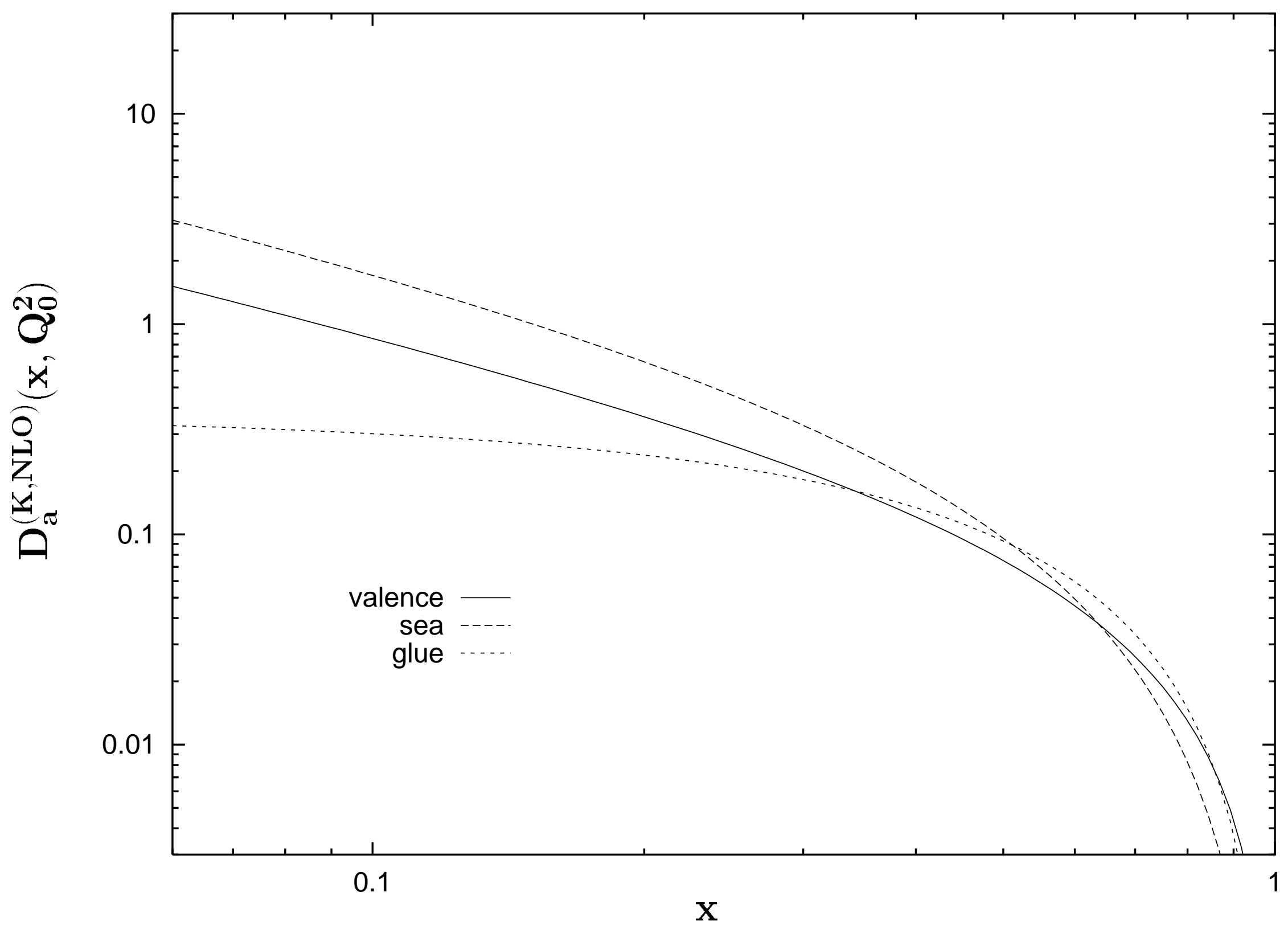

Fig. 2 


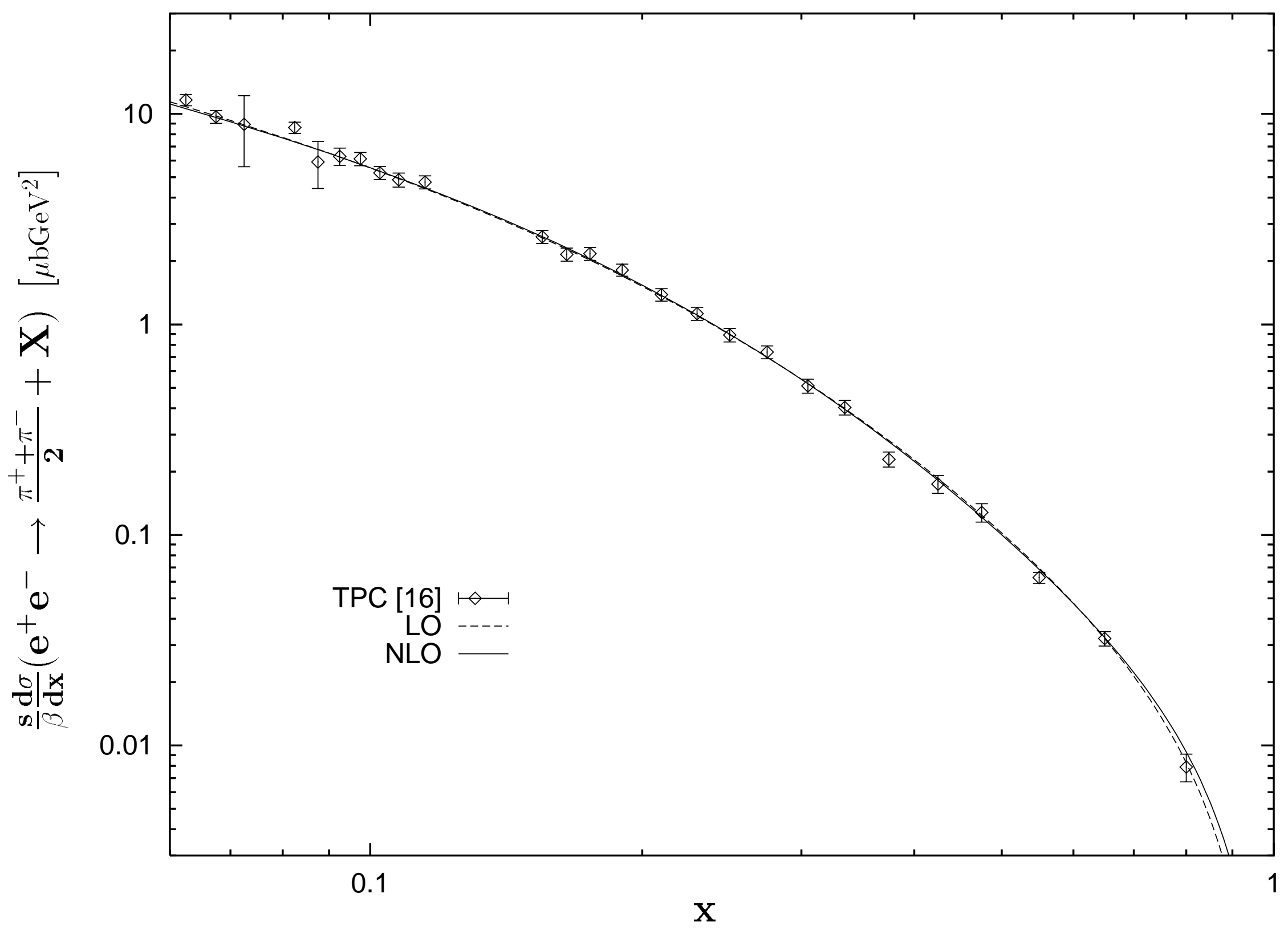

Fig. 3 


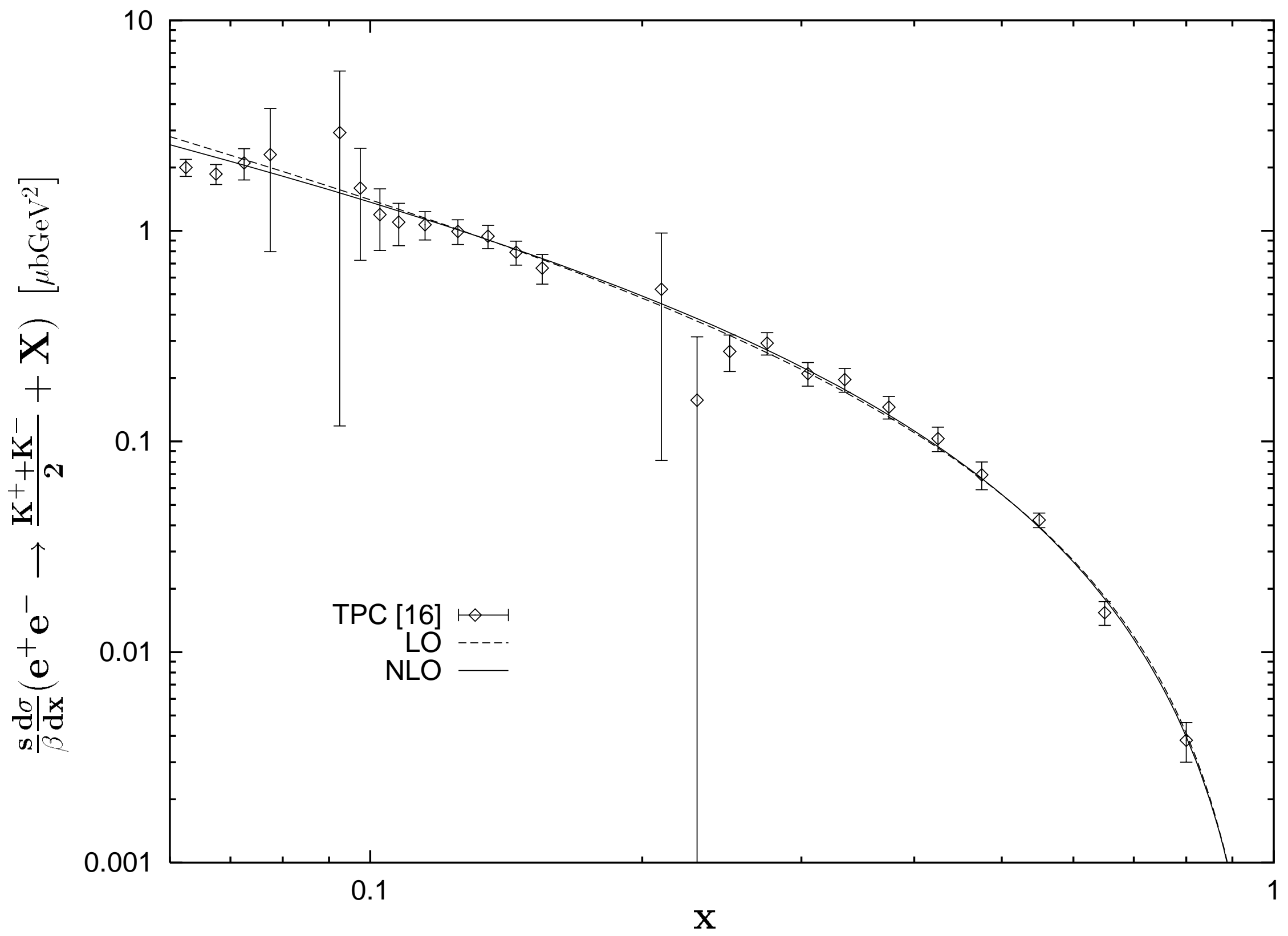

Fig. 4 


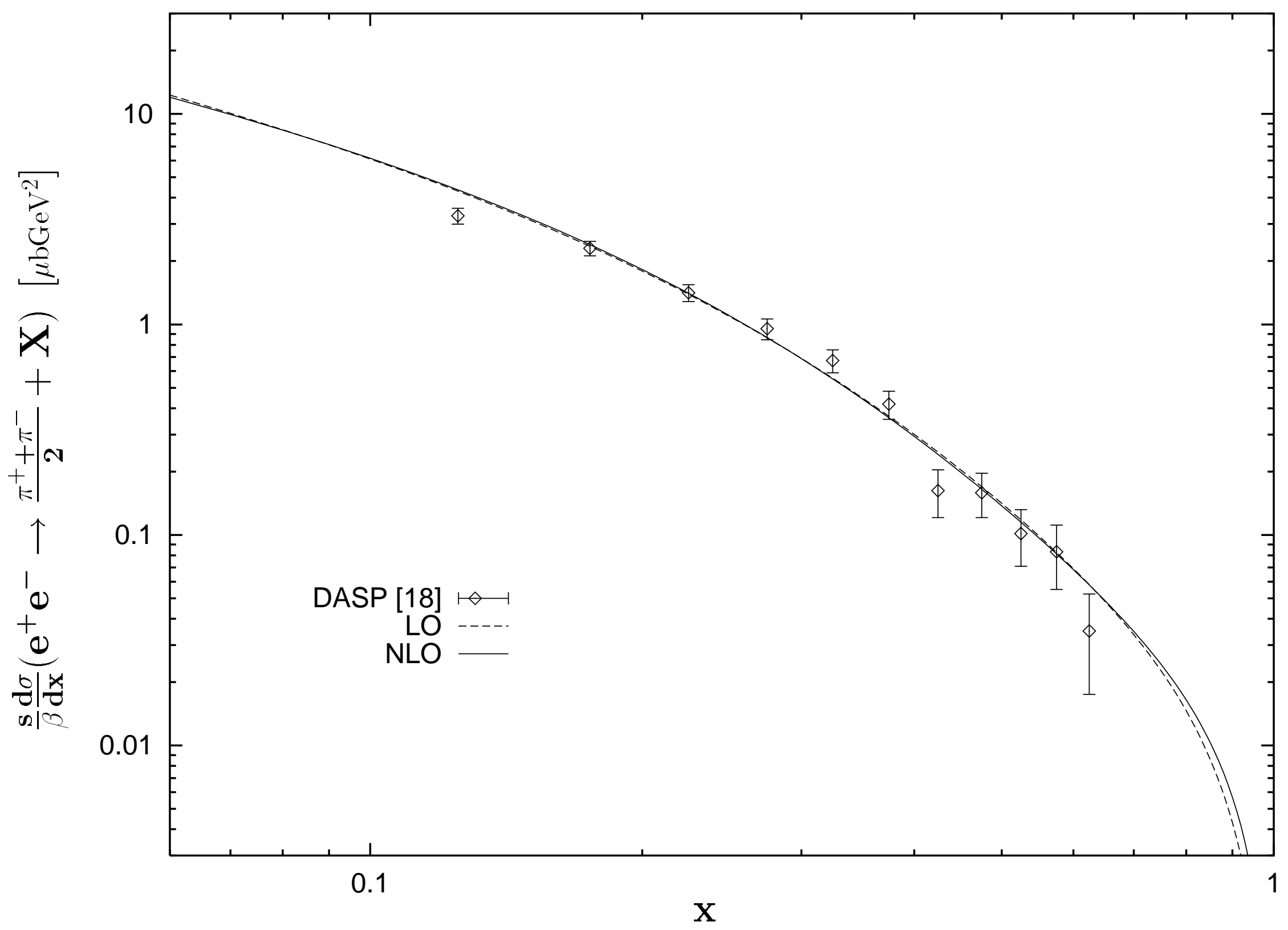

Fig. 5 


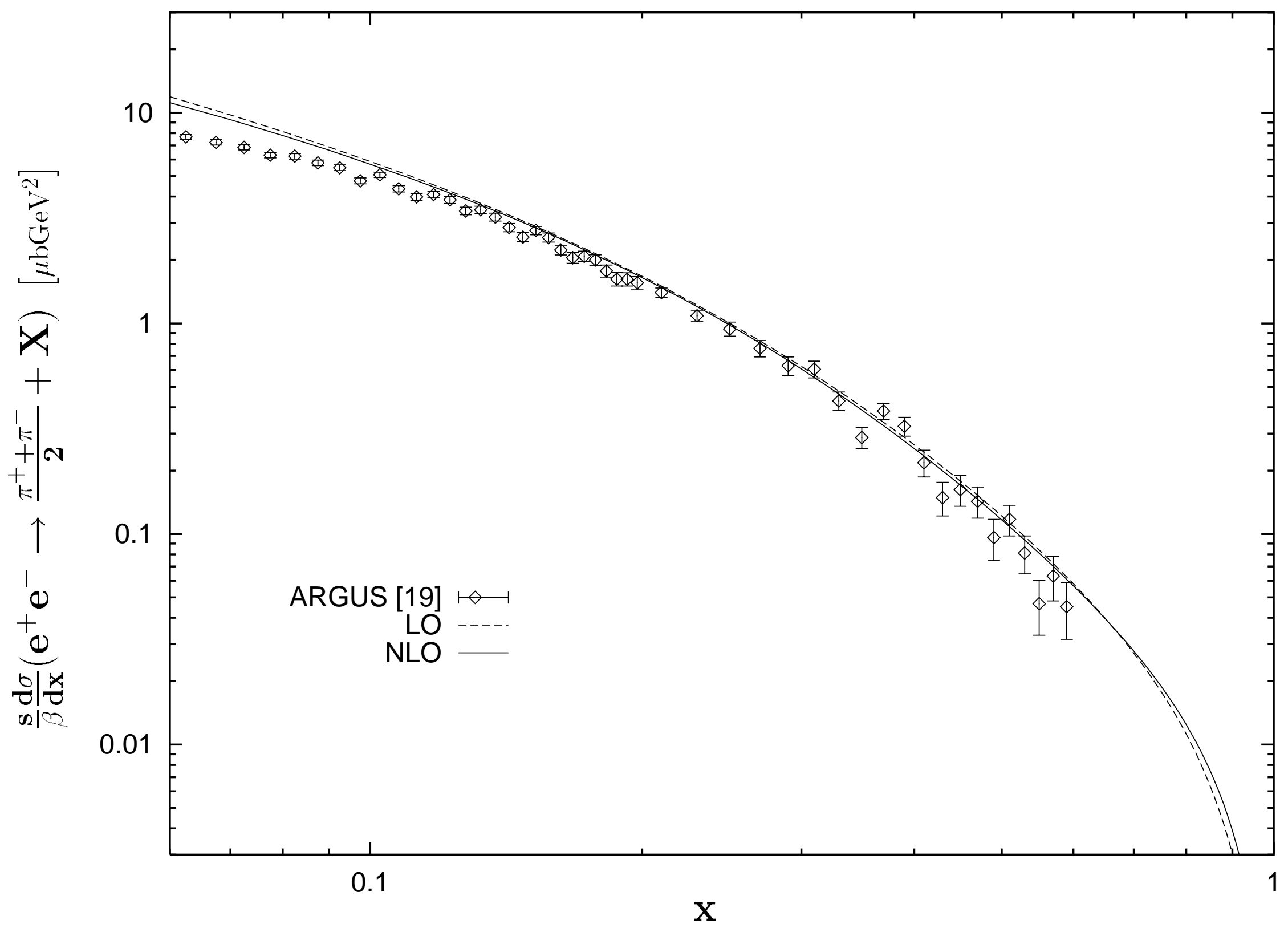

Fig. 6 


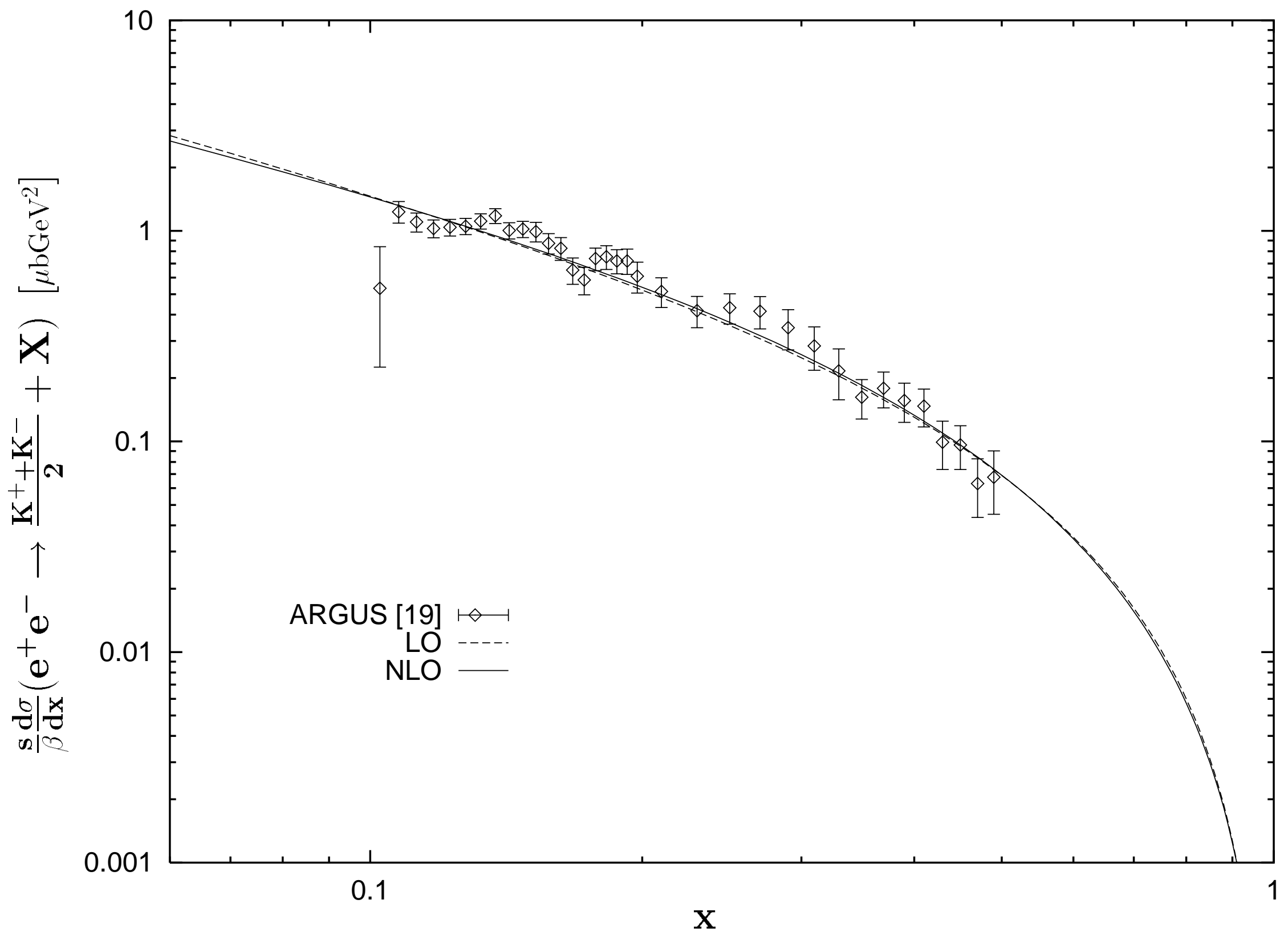

Fig. 7 


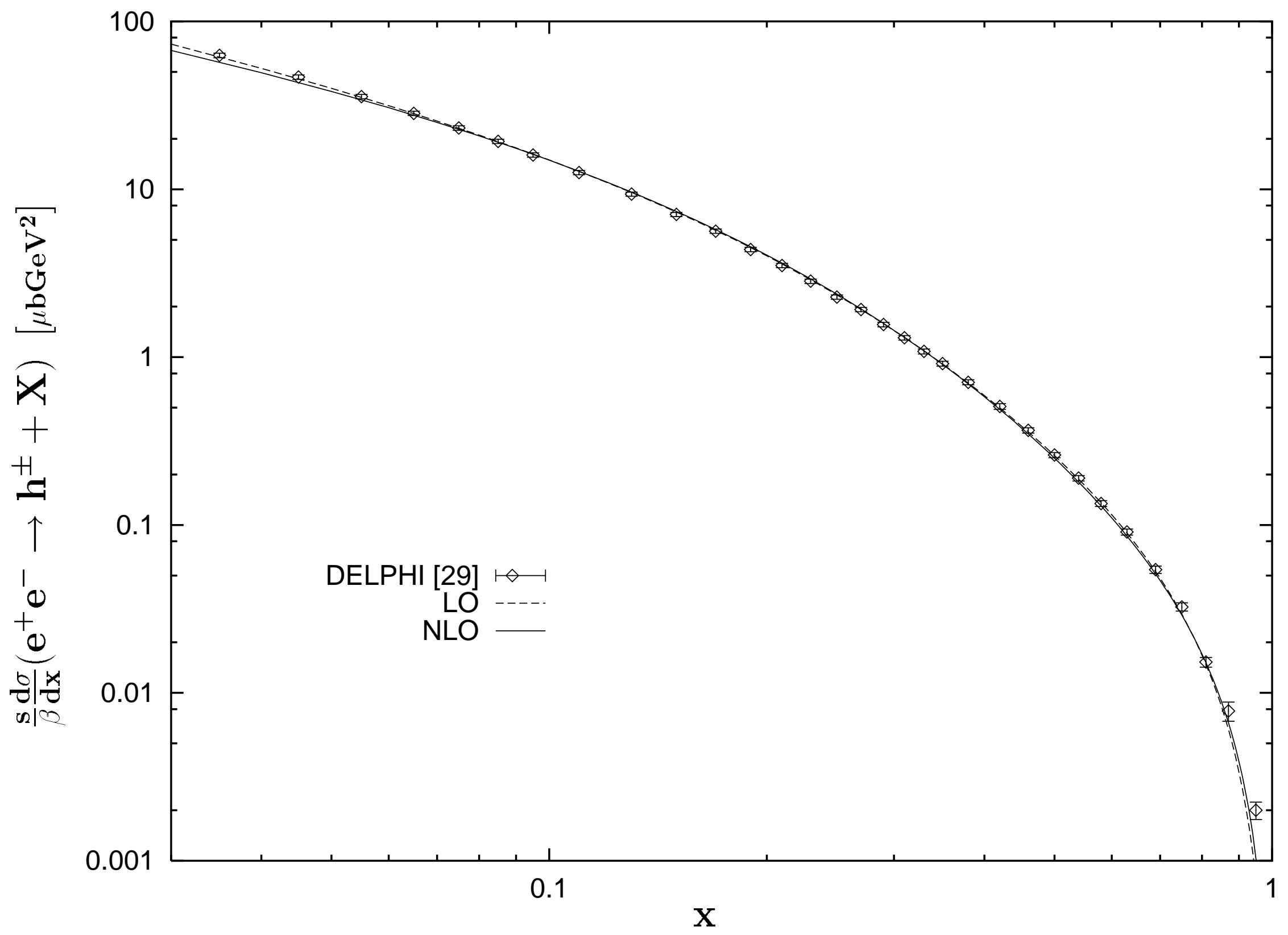

Fig. 8 


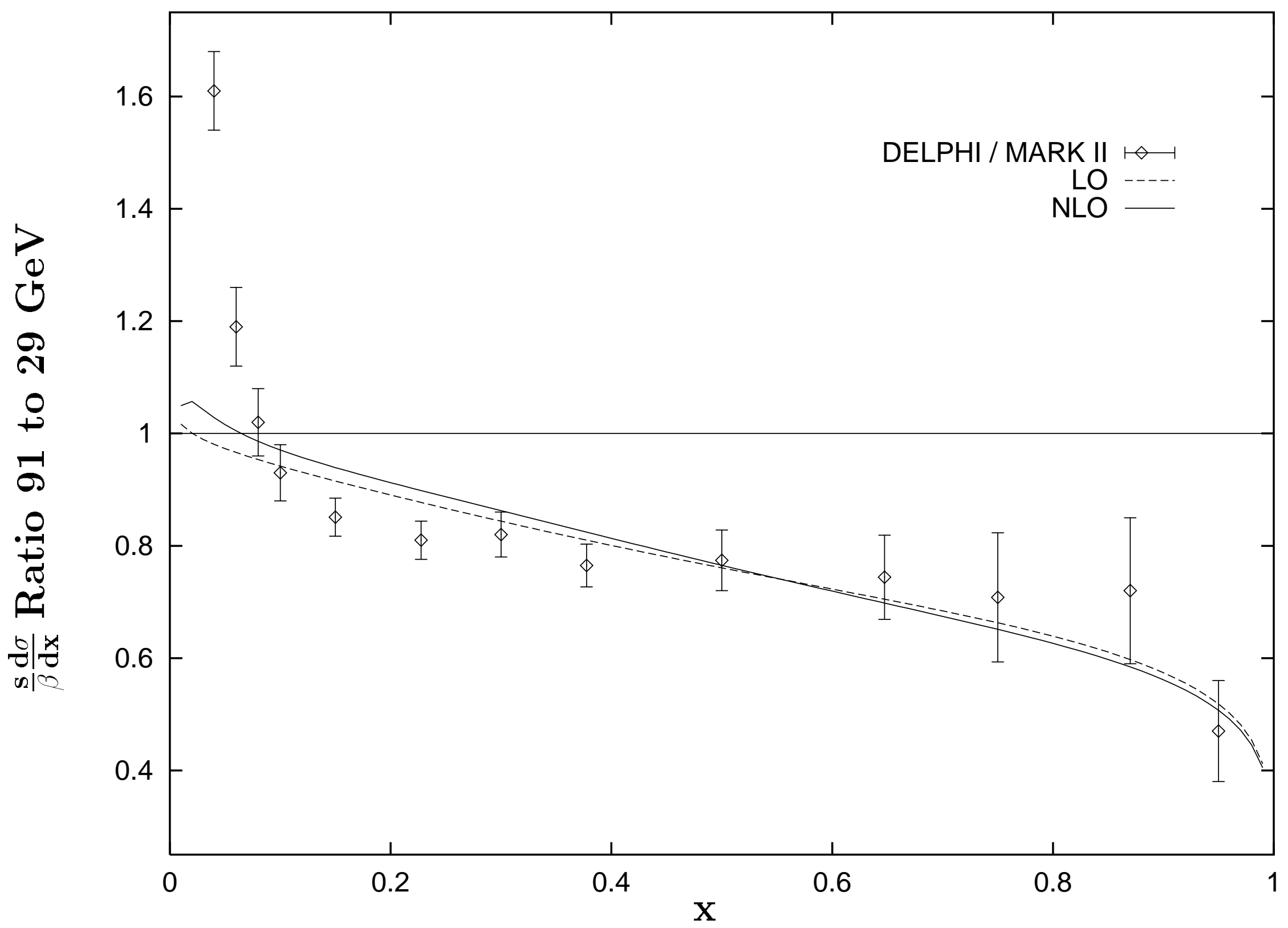

Fig. 9 


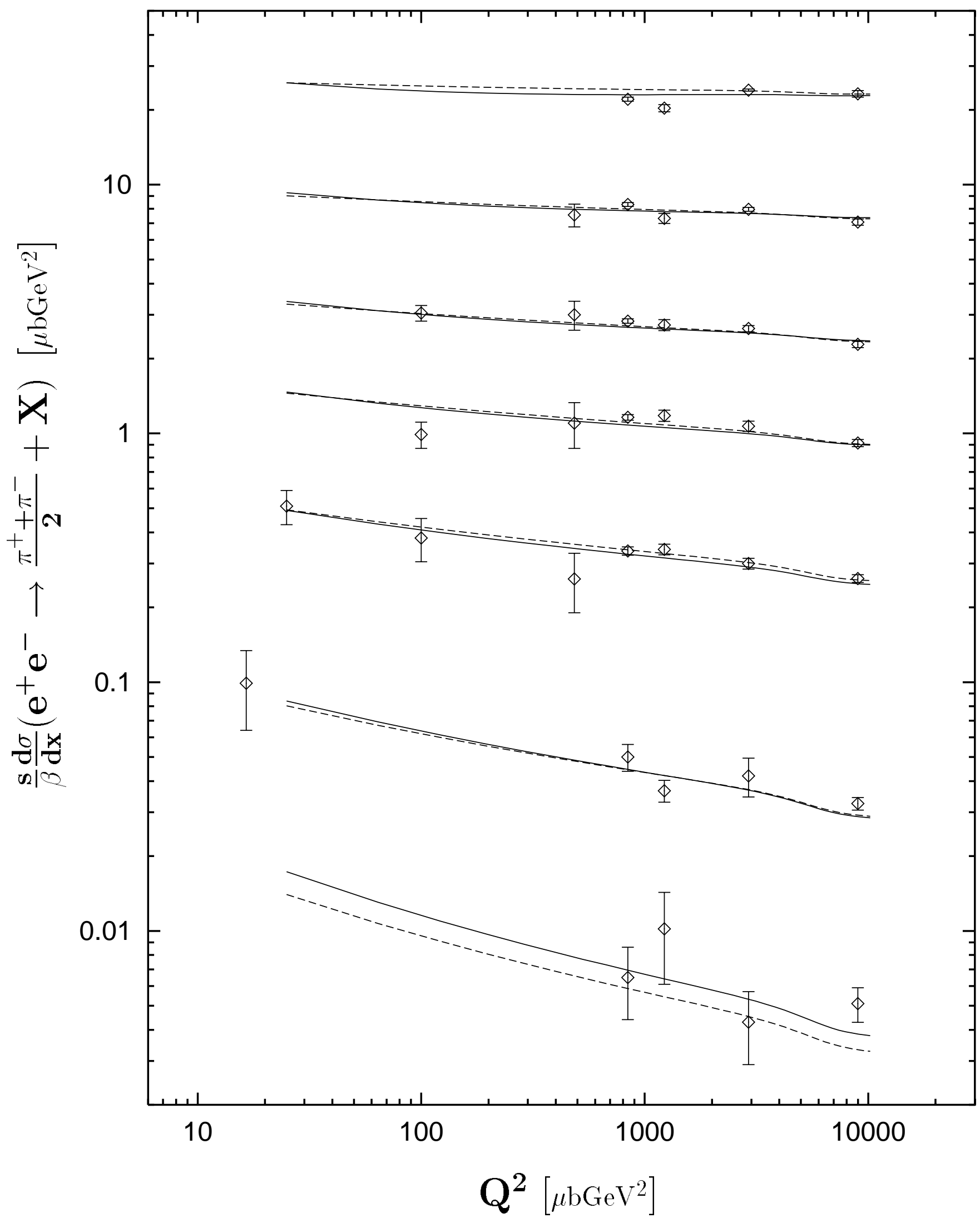

Fig. 10 


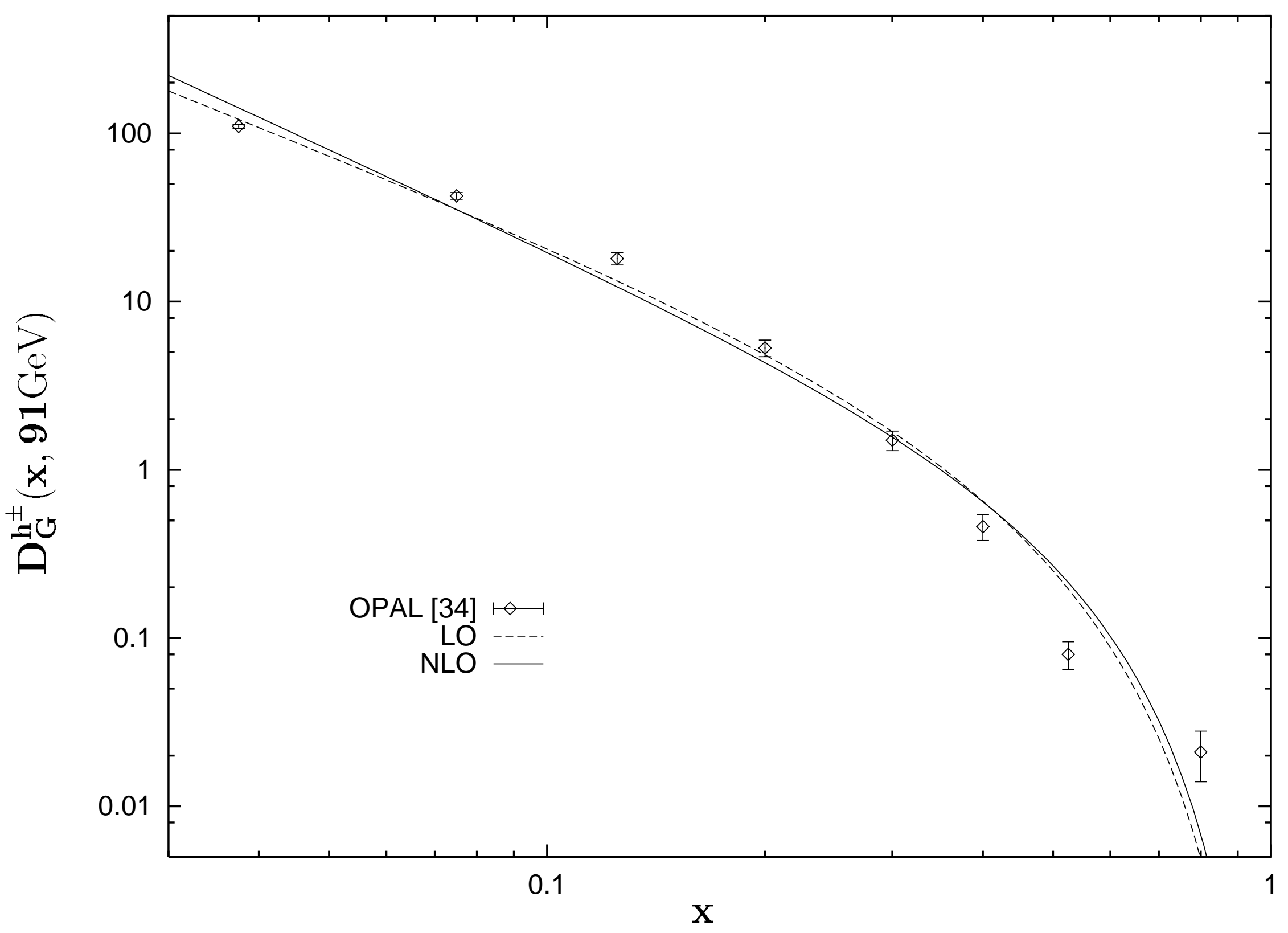

Fig. 11 